\title{
Determination of the heat transfer coefficient between bulk medium and packed containers in a batch retort
}

\author{
Szabolcs Varga ${ }^{\text {a }}$, Jorge C. Oliveira ${ }^{\text {b,* }}$ \\ ${ }^{a}$ Escola Superior de Biotecnologia, Universidade Católica Portuguesa, Rua Dr António Bernardino de Almeida, 4200 Porto, Portugal \\ ${ }^{\mathrm{b}}$ Department of Food Science and Technology, University College Cork, Cork, Ireland
}

\begin{abstract}
The external heat transfer coefficient in steam retort processing was determined experimentally in a pilot scale retort. The heat transfer equations were solved applying finite elements and using the actual retort temperature profile as boundary condition. The instantaneous values of the heat transfer coefficient were determined, to analyse its time-variability along a retort cycle. It was found that reliable results for the external heat transfer coefficient at time $t$ could be obtained from the derivatives of the average heat transfer coefficient calculated between time zero and time $t$. The results showed a sharp increase of the heat transfer coefficient in the earlier times of heating (up to 4-5 min of processing), followed by a slightly increasing pattern during the remaining heating period. Using average heat transfer coefficients for the heating phase (and for the cooling phase) also resulted in quite accurate estimates of the temperature at the geometric centre of a can. The influence of the two average heat transfer coefficients, heating and cooling, on the lethality was studied by building a response surface. It was concluded that the variability of the coefficient during heating has a greater impact, especially in the range $150-260 \mathrm{~W} / \mathrm{m}^{2} \mathrm{~K}$.
\end{abstract}

Keywords: Canning; Convection; Finite elements; Process variability; Sterilisation

\section{Introduction}

The design of the required thermal process for a given food product is affected by how the heat is transferred: (i) to the product and (ii) inside the food. The heat transfer inside the package depends on the properties of the food system and on the filling (headspace). The heat transfer from the retort heating or cooling medium to the product (surface of the containers) is determined by the heating medium temperature and the heat transfer coefficient.

The measurement of the relevant factors concerning heat transfer inside the container is relatively simple for conduction heating products, although more problematic for convection heating, and may vary from location to location, but does not usually vary with time (except when the physical properties change with cooking - e.g. gelation). The factors affecting the heat transfer from the retort heating medium (bulk) to the surface of the container may vary significantly with both location and

\footnotetext{
${ }^{*}$ Corresponding author. Tel.: +353-21-902748; fax: +353-21-276398
} E-mail address: j.oliveira@ucc.ie (J.C. Oliveira). time where the location variability is due to the nonuniformity of the hydrodynamic conditions. There is very limited information on how to deal with this multidimensional problem.

The heat transfer coefficient $(h)$ is often calculated with empirical correlations between dimensionless numbers. This approach is obviously restricted to the range of conditions for which the correlation was determined and to the design characteristics of the retort system (Dickerson \& Read, 1968; Anantheswaran \& Rao, 1985a,b; Merson \& Stophoros, 1990). The calculation of the heat transfer coefficient involves fitting some process equations to the experimental data, with $h$ being the only factor to determine by the regression. Recent work in mass transfer (Azevedo, Oliveira \& Drumond, 1998) concluded with statistical certainty that if both the internal and external heat transfer parameters (that is, $h$ and the thermal diffusivity $\alpha$ ) were to be determined jointly by experimental data, the errors would be very large and a strong collinearity between the parameters would prevent a minimally reasonable estimate of the parameters. An exception may occur in a relatively narrow intermediate range of the Biot number where the internal and external resistance are balanced. 
Therefore, the internal heat transfer must be well described by previous data before calculation of the external heat transfer resistance.

If the process is simplified, analytical solutions of Fourier's equations may be used to estimate the $h$ values from recorded temperature histories (Ling, Lund \& Lightfoot, 1976; Rumsey, Farkas \& Hudson, 1980; Naveh \& Kopelman, 1980; Ramaswamy, Tung \& Stark, 1983; McGinnis, 1986; Burfoot \& Self, 1988), otherwise it is necessary to solve the heat transfer problem numerically and determine $h$ by an optimisation method (Rumsey et al., 1980; Burfoot \& James, 1988; Chang \& Toledo, 1989; Lebowitz \& Bhowmik, 1989, 1990; Xie \& Sheard, 1996). These calculations have not been applied to situations where $h$ may vary during the whole sterilisation cycle. Ling et al. (1976) used an analytical solution to calculate $h$ with time for a spherical particle. Rumsey et al. (1980) reported results on estimating the heat transfer coefficient at the surface of potato cylinders by solving the overall heat balance using both Laplace Transformation and a finite difference numerical model. A short process time of $60 \mathrm{~s}$ was considered, and the results indicated an initial decrease of $h$ with time, in some cases, approaching a constant value after $20 \mathrm{~s}$ of processing.

The objective of this work was to determine experimentally the overall heat transfer coefficient describing the heat transfer from the heating and cooling mediums of a retort to the inner surface of packed containers in fully loaded retorts, analysing whether by numerical modelling it would be possible to obtain sufficient information to calculate a time varying heat transfer coefficient. The impact on the lethality achieved in a conduction-heating product was also to be studied.

\section{Materials and methods}

\subsection{Experimental set-up}

A $10 \%$ bentonite suspension was filled into cans of $3.65 \mathrm{~cm}$ radius and $10.3 \mathrm{~cm}$ height with $8 \%$ headspace. Three layers of nine cans containing water were placed in a Barriquand Steriflow pilot scale retort in a squared layout. Two cans with the bentonite solution were equipped with Ellab thermocouples along the horizontal centre line so that the temperature sensor touched the can wall inside the suspension. One can was placed in the centre of the middle layer and the other one at the corner of the top layer, as these were judged to be extreme conditions. To measure the heating medium temperature, two thermocouples were placed near the cans (approximately $5 \mathrm{~cm}$ from the surface) in the direction of the internal ones. The racking layouts used are shown in Fig. 1.

The heating medium was saturated steam. The comeup was followed by a $40 \mathrm{~min}$ holding phase at $126^{\circ} \mathrm{C}$.

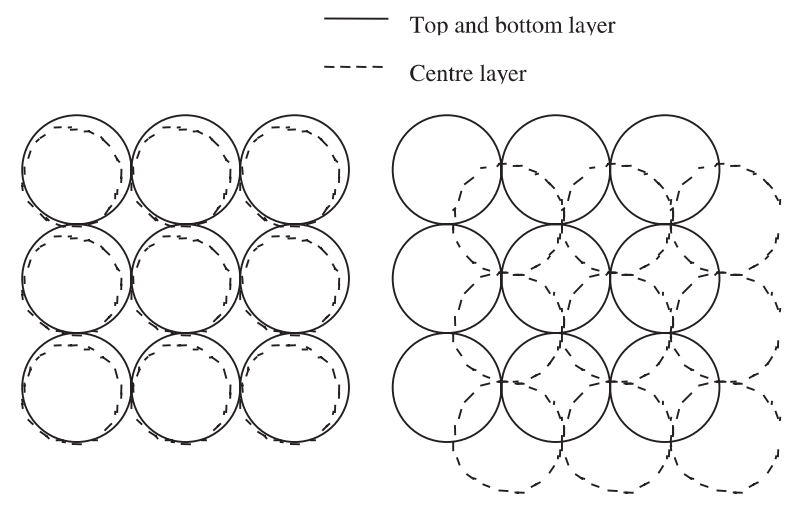

a)

b)

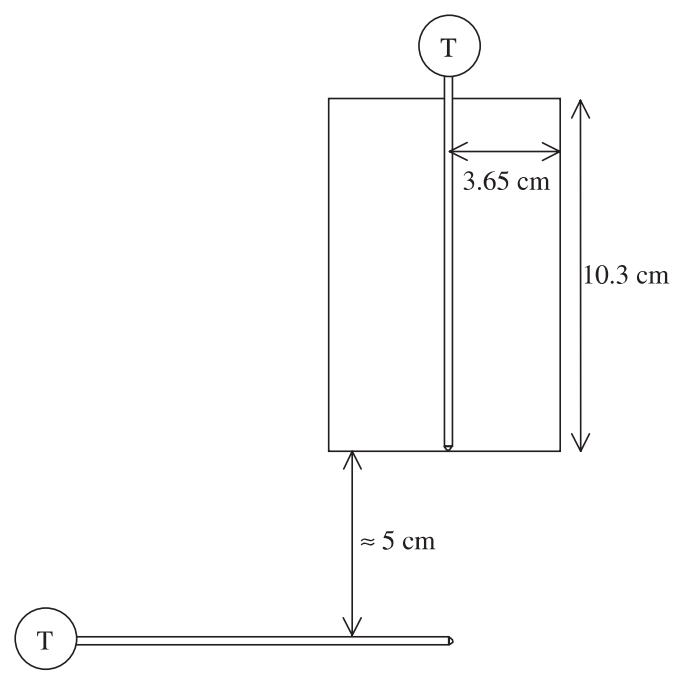

c)

Fig. 1. Racking layout: (a) racking layout a; (b) racking layout b; (c) position of thermocouples.

Tap water was used for cooling. Four experiments were performed, two with layout a and two with $b$ (see Fig. 1). The temperature data were collected every $15 \mathrm{~s}$.

\subsection{Solution of the heat transfer equations}

The well known Fourier's second law for finite cylinder geometry describes the problem mathematically for a conduction-heating material inside the container

$\frac{\partial T}{\partial t}=\alpha\left(\frac{\partial^{2} T}{\partial z^{2}}+\frac{1}{r} \frac{\partial^{2}(r T)}{\partial r^{2}}\right)$

with the limit conditions:

Initial condition:

$t=0, \quad T=T_{0} \quad \forall r, z$,

Symmetry condition:

$r=0, \quad \frac{\partial T}{\partial r}=0 \quad \forall t$ 
Boundary conditions:

$$
\begin{aligned}
& \text { (a) } r=R \quad-\left.k \frac{\partial T}{\partial r}\right|_{r=R}=h_{\mathrm{t}}\left(T(t)_{\text {surf }}-T(t)_{\infty}\right), \\
& \text { (b) } z=0, H \quad-\left.k \frac{\partial T}{\partial z}\right|_{z=0, H}=h_{\mathrm{t}}\left(T(t)_{\text {surf }}-T(t)_{\infty}\right) .
\end{aligned}
$$

The heat conduction problem was solved in ANSYS5.3 (ANSYS, Houston, USA) using the experimental surface temperature as constraints of the boundary nodes (boundary conditions). Some details on the solution of the heat transfer problem with finite elements can be found elsewhere (Varga, Oliveira, Smout \& Hendrickx, 2000). The can was divided into 64 four node rectangular axisymmetric elements with decreasing volume in the direction of the boundary in order to improve accuracy. The $10 \%$ bentonite suspension had density of $1070.5 \mathrm{~kg} \mathrm{~m}^{-3}$, specific heat $3866 \mathrm{~J} \mathrm{~kg}^{-1}{ }^{\circ} \mathrm{C}^{-1}$ and thermal conductivity of $0.7754 \mathrm{~W} \mathrm{~m}^{-1}{ }^{\circ} \mathrm{C}^{-1}$.

The temperature at any time at any location inside the container can be expressed as a function of the nodal temperatures

$T=N_{i} T_{i}+N_{j} T_{j}+N_{k} T_{k}+N_{m} T_{m}$,

where

$$
\begin{aligned}
& N_{i}=\frac{1}{4 a b}\left(R_{j}-r\right)(H-h), \\
& N_{j}=\frac{1}{4 a b}\left(r-R_{i}\right)(H-h), \\
& N_{k}=\frac{1}{4 a b}\left(r-R_{i}\right) h, \\
& N_{m}=\frac{1}{4 a b}\left(R_{j}-r\right) h,
\end{aligned}
$$

$N$ is called the element shape function. Subscript $i$ corresponds to the node of an element nearest to the origin of the co-ordinate system and the other letters are assigned counter clockwise. $H$ is the height of the element.

The element average temperature $\left(T_{\text {Eav }}\right)$ is then obtained by integrating Eq. (2) over the element volume

$$
\begin{aligned}
T_{\text {Eav }}= & \frac{1}{V_{\mathrm{E}}} \int_{\mathrm{Vol}} T \mathrm{~d} V=\frac{2 \pi}{V_{\mathrm{E}}} \int_{0}^{H} \int_{R_{i}}^{R_{j}} T \mathrm{~d} r \mathrm{~d} h \\
= & \frac{1}{\left(R_{j}-R_{i}\right)\left(R_{j}^{2}-R_{i}^{2}\right)}\left[\left(\frac{R_{i}^{3}}{3}-\frac{R_{i}^{2} R_{j}}{2}+\frac{R_{j}^{3}}{6}\right)\right. \\
& \left.\times\left(T_{i}+T_{m}\right)+\left(\frac{R_{i}^{3}}{6}-\frac{R_{i} R_{j}^{2}}{2}+\frac{R_{j}^{3}}{3}\right)\left(T_{j}+T_{k}\right)\right] .
\end{aligned}
$$

The volume average temperature $\left(T_{\text {ave }}\right)$ is then obtained

$T_{\mathrm{ave}}=\frac{\sum_{i=1}^{N} T_{\mathrm{Eav}}^{i} V_{\mathrm{E}}^{i}}{V}$.

The differential term of $T_{\text {ave }}$ was calculated with a numerical subroutine in ANSYS 5.3. The fact that the surface temperature was measured from the inside, just touching the can surface, implies that the calculated heat transfer coefficient involves the external film, the effect of the resistance of the can wall and of a likely thin film of the bentonite suspension (around the tip of the thermocouple). Therefore, it can be considered as an overall heat transfer coefficient. It was found by mathematical modelling that the effect of a $1 \mathrm{~mm}$ layer of bentonite solution could be considered negligible and therefore the thermocouple reading is taken as a measurement of the inner temperature of the can wall. The application of an overall $h$ in mathematical models for predicting product temperature is consistent, since most models neglect the effect of the container wall and the wall/product heat transfer.

\subsection{Determination of time-varying heat transfer coeffi- cients}

It might be thought that the instantaneous value of the heat transfer coefficient $\left(h_{\mathrm{t}}\right)$ could be simply obtained by solving the overall heat balance equation (Ling et al., 1976)

$\rho C_{\mathrm{p}} V \frac{\mathrm{d} T_{\text {ave }}^{t}}{\mathrm{~d} t}=h_{\mathrm{t}} A\left(T_{\infty}^{t}-T_{\text {surf }}^{t}\right)$,

where $T_{\infty}, T_{\text {surf }}$ and $T_{\text {ave }}$ are the heating medium and the product surface and volume average temperatures, respectively (the superscript $t$ indicates that they are functions of time). However, this would imply determining values of $h_{\mathrm{t}}$ out of very small heat fluxes in the numerical calculations, corresponding to the incremental time steps into which the domain was discretized by the numerical procedure. The resulting situation is similar to trying to solve Eq. (5) for small temperature gradients - if $\Delta t$ or $\Delta T$ are very small, the error of the determination of $h$ is obviously very high. Notice is made of this point as it is possible to find in literature works where this error was not noticed (Hubbard \& Farkas, 1998). Normally, the result is to find instantaneous $h$ values that vary pretty much in the inverse manner of the heat flux intensity and that are very high.

The alternative to avoid calculating $h$ values for very small heat fluxes (time steps) is to determine the average heat transfer coefficient $\left(\bar{h}_{\mathrm{t}}\right)$ between time zero and time $t$, as by definition

$\bar{h}_{\mathrm{t}}=\frac{\int_{t_{0}}^{t} h_{\mathrm{t}} \mathrm{d} t}{t-t_{0}}$,

where $t_{0}$ is the initial time for the retort cycle phase being considered ( 0 for heating). The instantaneous coefficient at time $t\left(h_{\mathrm{t}}\right)$ can be calculated by derivating Eq. (2)

$h_{\mathrm{t}}=\bar{h}_{\mathrm{t}}+\left(t-t_{0}\right) \frac{\mathrm{d} \bar{h}_{\mathrm{t}}}{\mathrm{d} t}$. 


\subsection{Determination of average heat transfer coefficients}

The average heat transfer coefficient from $t_{0}$ to the several values of $t$ considered can be calculated by two ways:

(a) Integrating the heat balance (Eq. (5)):

$$
\begin{aligned}
\bar{h}_{\mathrm{t}} & =\frac{\rho c_{\mathrm{p}} V}{A} \frac{\int_{T_{0}}^{T_{\text {ave }}^{t}} \mathrm{~d} T_{\mathrm{ave}}}{\int_{0}^{t}\left(T_{\infty}^{t}-T_{\text {surf }}^{t}\right) \mathrm{d} t} \\
& =\frac{\rho c_{\mathrm{p}} V}{A} \frac{T_{\mathrm{ave}}^{t}-T_{0}}{\int_{0}^{t}\left(T_{\infty}^{t}-T_{\text {surf }}^{t}\right) \mathrm{d} t} .
\end{aligned}
$$

(b) Determining the value of $h$ that minimises the difference between experimental and model temperatures, by optimising the residual sum of squares (SSR).

The latter is a more widely used technique, but in most literature cases a constant medium temperature is considered. In this work, the experimental retort time temperature profile was used as time dependent boundary condition in the conduction heating finite element model. The ANSYS 5.3 first order design optimisation method was used to find the values of $h$ that result in the minimum value of the residual sum of squares (SSR)

$\mathrm{SSR}=\sum\left(T_{\text {experimental }}^{x, r, t}-T_{\text {simulated }}^{x, r, t}\right)^{2}$.

The average $h$ values for the whole heating period (up to holding) and for the cooling phase were designated specifically as $h_{\text {heat }}$ and $h_{\text {cool }}$, respectively. The superscripts in Eq. (9) indicate that the location of the temperature measurement must be known, but it can be anywhere in the container. The data should be compared to the simulated values exactly at the same point. The selection of a location at the surface means that the

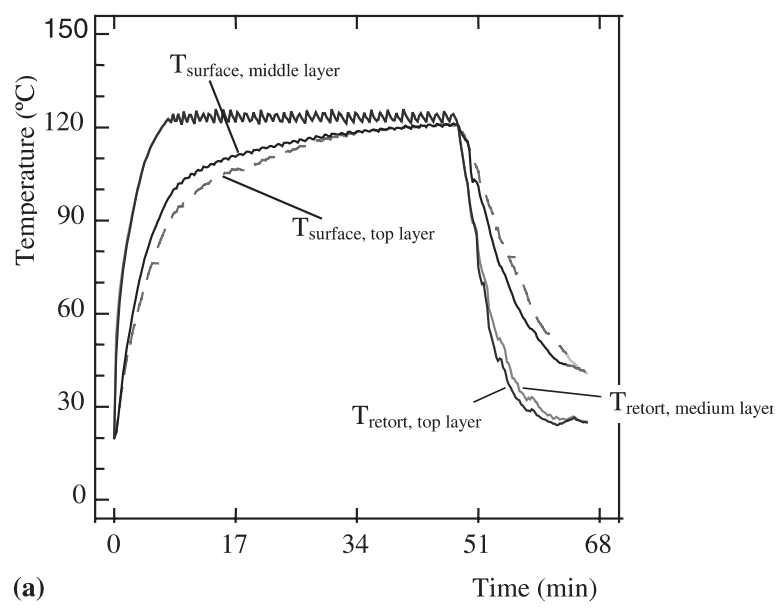

sensitivity of the system is larger than using an interior point $\left(\partial S S R / \partial h_{\text {surf }}>\partial S S R / \partial h_{\text {interior }}\right)$. This also implies that when using the temperature measured at the surface a small error in the location of the probe results in a larger error in the $h$ value, compared to the use of the centre temperature. The latter also has the disadvantage that the internal heat transfer must be well described, that is, the properties of the material inside the container must be accurately known.

The first order optimisation method used in this work involves the formulation of the constrained objective function (Eq. (9)) to unconstrained by applying penalty functions to the design variable $\left(\bar{h}_{\mathrm{t}}\right)$. The search direction was determined by the steepest descent method using the derivatives of the objective function. Further details can be found in the ANSYS 5.3 reference manuals.

\section{Results and discussion}

Both procedures for determining the average heat transfer coefficient resulted in virtually identical values, but procedure (b) (minimisation of the SSR between simulated and experimental temperatures) obviously requires much more computational time, and so procedure (a) (application of Eq. (8)) is preferable.

Typical recorded time-temperature profiles are shown in Fig. 2(a) and (b) for the two racking layouts, respectively (first batch in both cases). Fig. 3(a) and (b) give the instantaneous values of $h_{\mathrm{t}}$ calculated in the heating phase. The average heat transfer coefficients for heating and cooling are given in Table 1.

In each experiment, regardless of the racking layout, the surface of the can in the middle layer heated faster than in the top layer. Fig. 3 shows that for both racking layouts, the middle layer had slightly higher heat

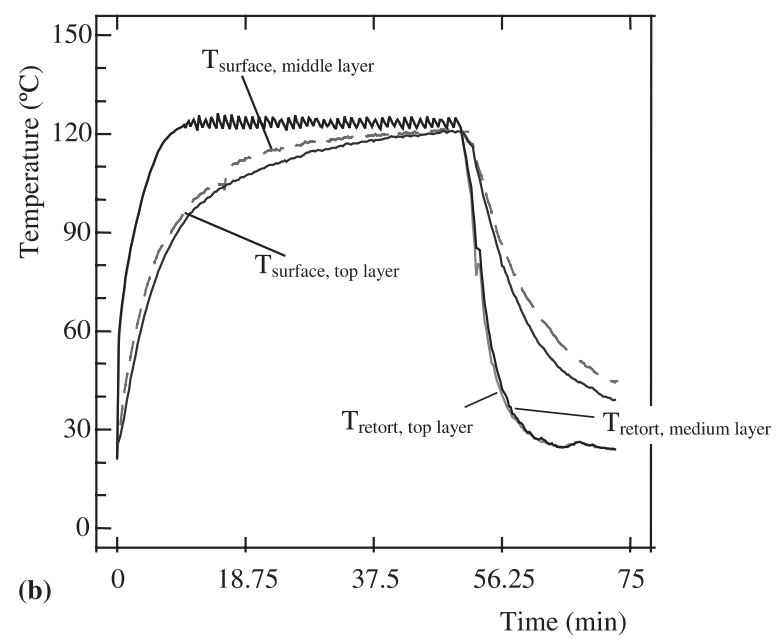

Fig. 2. Temperature data for cans filled with bentonite solution in two replicate batches: (a) racking layout a; (b) racking layout b. 

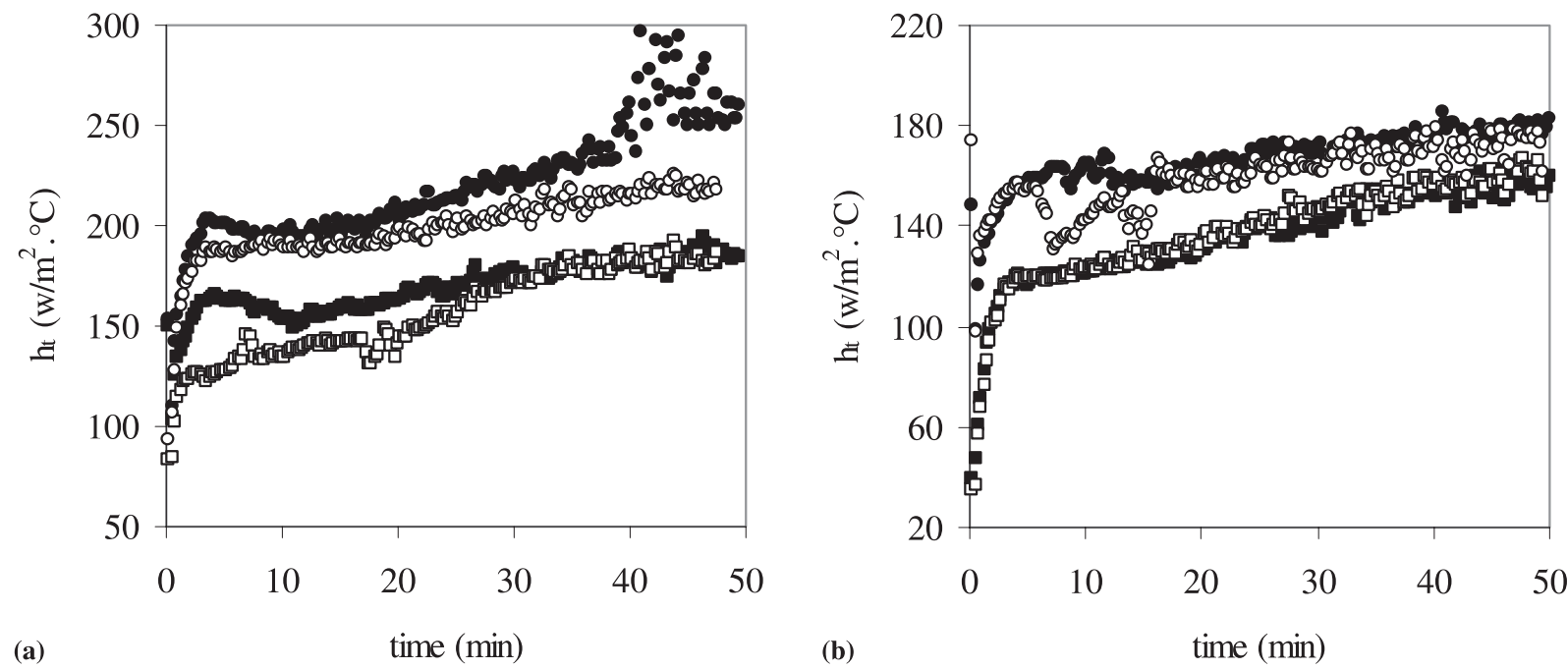

Fig. 3. Instantaneous heat transfer coefficients at several processing times in two replicate batches: (a) racking layout a; (b) racking layout b; (O) first batch, middle layer; $(\bullet)$ second batch, middle layer; $(\square)$ first batch, top layer; ( $\square$ ) second batch, top layer.

Table 1

Calculated average $h$ values $\left(\mathrm{W} \mathrm{m}^{-2}{ }^{\circ} \mathrm{C}^{-1}\right)$

\begin{tabular}{lcc}
\hline Racking/replicate & Middle & Top \\
\hline Heating & 190 & \\
a/1 & 203 & 146 \\
a/2 & 189 & 164 \\
b/1 & 130 \\
b/2 & 162 & 129 \\
Cooling & & \\
a/1 & 125 & 100 \\
a/2 & 142 & 109 \\
b/1 & 75 & 95 \\
b/2 & 121 & 100 \\
\hline
\end{tabular}

transfer coefficients, which explains the slightly higher heating rate. The physical explanation for this may be the higher steam circulation velocity in the space between cans due to the lower cross sectional flow area, thereby indicating that with both racking layouts used the steam within cans is not stagnant, but flowing (otherwise, the middle layer would heat slower). The second racking layout has slightly lower heat transfer coefficients, which may again be related to this channelling effect that improves heat transfer in the space between cans. As the layers in the second racking layout are not aligned, the channelling flow is hindered. Fig. 3 also shows that there are differences between batches, though mostly small and in some cases even negligible, and that these are much smaller than differences between layers in a given batch.

The instantaneous heat transfer coefficient increases sharply in the first few minutes, reaching a value close to the average in less than $5 \mathrm{~min}$. This increase may be due to the air that is still present until complete venting out, as the air is non-condensable and the condensation near

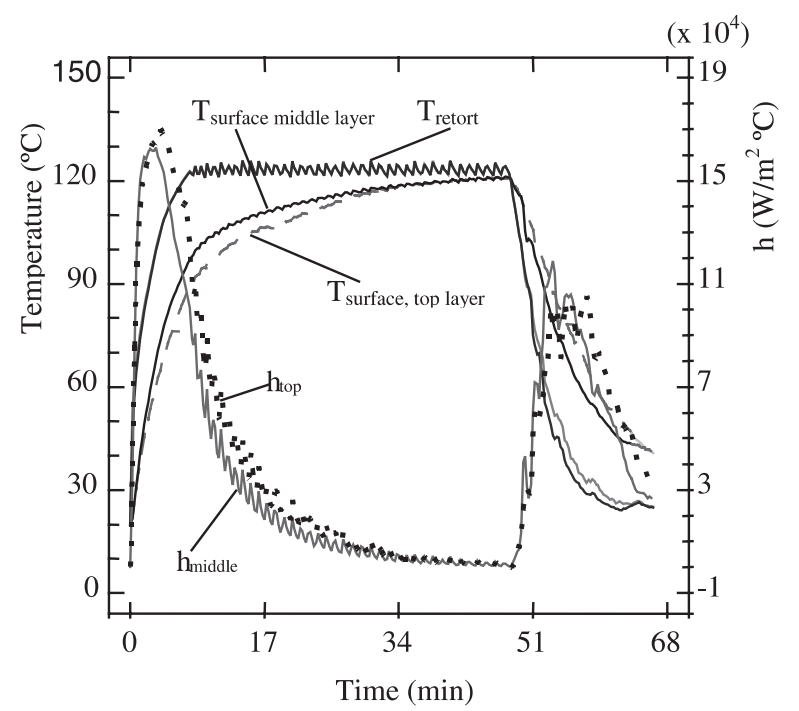

Fig. 4. Instantaneous heat transfer coefficients that would be calculated by Eq. (5) for the first batch with racking layout a.

the can wall improves heat transfer. Subsequently, the heat transfer coefficient rises slightly. The average heat transfer coefficients were higher in the heating than in the cooling phase (which again may be due to the improved heat transfer resulting from condensation near the can walls).

Just out of curiosity, in order to strengthen the point on the calculation of $h$ factors with low heat fluxes, Fig. 4 shows an example of the $h_{\mathrm{t}}$ values that would be calculated by direct application of Eq. (5). As stated previously, one then finds very high values of $h$ (these would not be acceptable physically, as they would imply virtually no external resistance, which Fig. 2 clearly shows that it is not the case), and furthermore, one would conclude that $h_{\mathrm{t}}$ tends to zero as the temperature 
gradient tends to zero - this is a clear indication of the error of these calculations.

As there is limited data on literature concerning heat transfer coefficients in retort processing, the average values obtained in our work (Table 1) were compared to a variety of situations. In general, they are low compared to film coefficients reported for different environments. Burfoot and Self (1988) reported high $h$ values $\left(830-1550 \mathrm{~W} / \mathrm{m}^{2} \mathrm{~K}\right)$ for meat cubes during cooking. Rumsey et al. (1980) indicated that $h$ approximated about $500 \mathrm{~W} / \mathrm{m}^{2} \mathrm{~K}$ for potato cylinders after a few seconds of blanching. McGinnis (1986) presented graphs of $h$ vs. heating and cooling medium temperature in a retort, where the heat transfer coefficient varied between $700-3000 \mathrm{~W} / \mathrm{m}^{2} \mathrm{~K}$ for plastic pouches. Significantly higher $h\left(\approx 5000 \mathrm{~W} / \mathrm{m}^{2} \mathrm{~K}\right)$ was obtained by Ramaswamy and Tung (1990) for a brick shape body in a water immersion retort. Tucker and Holdsworth (1991) also obtained an overall $h$ of $500 \mathrm{~W} / \mathrm{m}^{2} \mathrm{~K}$ for a rectangular package and condensing steam heating medium. Similar heat transfer coefficients to those shown in Table 1 were obtained by Chang and Toledo (1989) in a continuously flowing system, Xie and Sheard (1996) in a combination oven, and Lebowitz and Bhowmik (1989, 1990) in a modified still retort for cube, cylinder and pouch shape bodies - the cylinder case being the most similar to the one analysed in our work. The $h$ values of Table 1 are therefore within an acceptable range.

An explanation to the relatively low $h$ might be that due to the experimental set-up, the calculated heat transfer coefficients are global, not just external, and also include the thermal resistance of the can wall. A layer of bentonite suspension around the tip of the thermocouple could also interfere. However, model calculations indicated that the deviations caused by these two factors are small - generally less than $5 \%$. It is also possible that due to its small size, the circulation of steam in the retort was relatively limited, compared to industrial scale retorts. Low values of $h$ are however particularly important in water cascading retorts and so this range deserves some further analysis. Model calculations for our system indicated that heat transfer coefficients above $1000 \mathrm{~W} / \mathrm{m}^{2}{ }^{\circ} \mathrm{C}$ would have negligible impact on lethality.

\subsection{Influence of the external heat transfer coefficient on lethality}

The time-temperature profiles at the product surface calculated with the optimised average heat transfer coefficient and the instantaneous heat transfer coefficients are indistinguishable and generally fall on top of the experimental values. Fig. 5 shows the case where a larger difference between model and experimental values was found, with the model values calculated with average heat transfer coefficients for heating and for cooling. Therefore, it can be said that the use of an instantaneous heat transfer coefficient would not be really needed in this case - an average value for the whole heating period is good enough. However, it was found that it was better to separate the heating and the cooling phases that to use a single $h$ value for both phases, which is not surprising, since the media are different (steam in heating, liquid water in cooling).

The $F$-value was then calculated as a function of $h_{\text {heat }}$ and $h_{\text {cool }}$ for the geometric centre of a can containing bentonite (conduction heating). The heating medium temperature history of batch 1 was used as boundary conditions in the numerical solution. A surface plot of the results is shown in Fig. 6. Higher values of the heat transfer coefficients were not tested, as in those conditions the external resistance would be negligible.

It can be seen that the higher the $h_{\text {heat }}$ and the lower the $h_{\text {cool }}$ the higher the lethality and that generally the

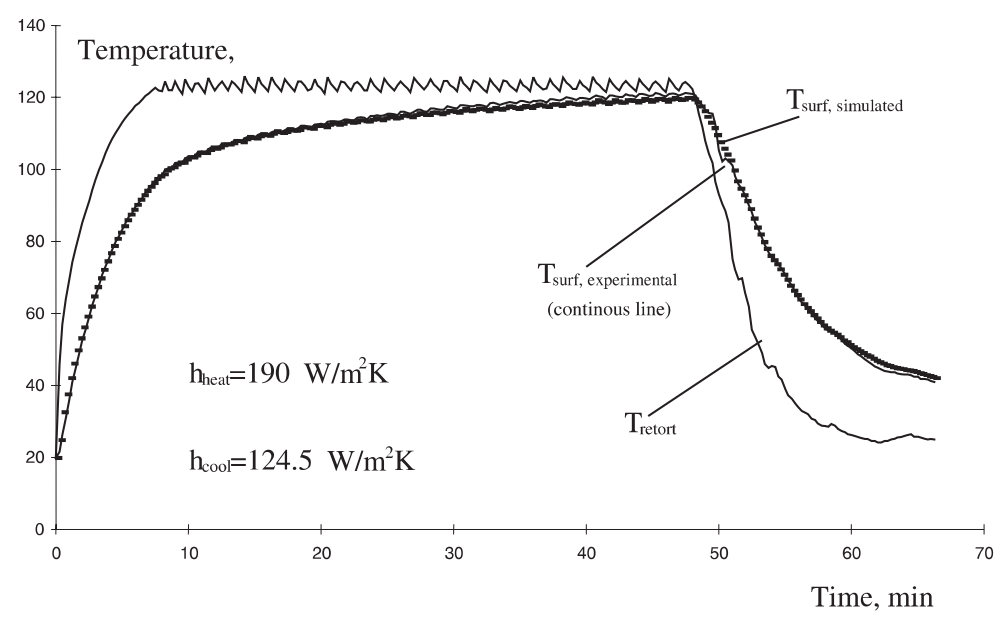

Fig. 5. Comparison of model and experimental temperatures at the product surface. Model values were calculated with average heat transfer coefficients for heating and for cooling. 


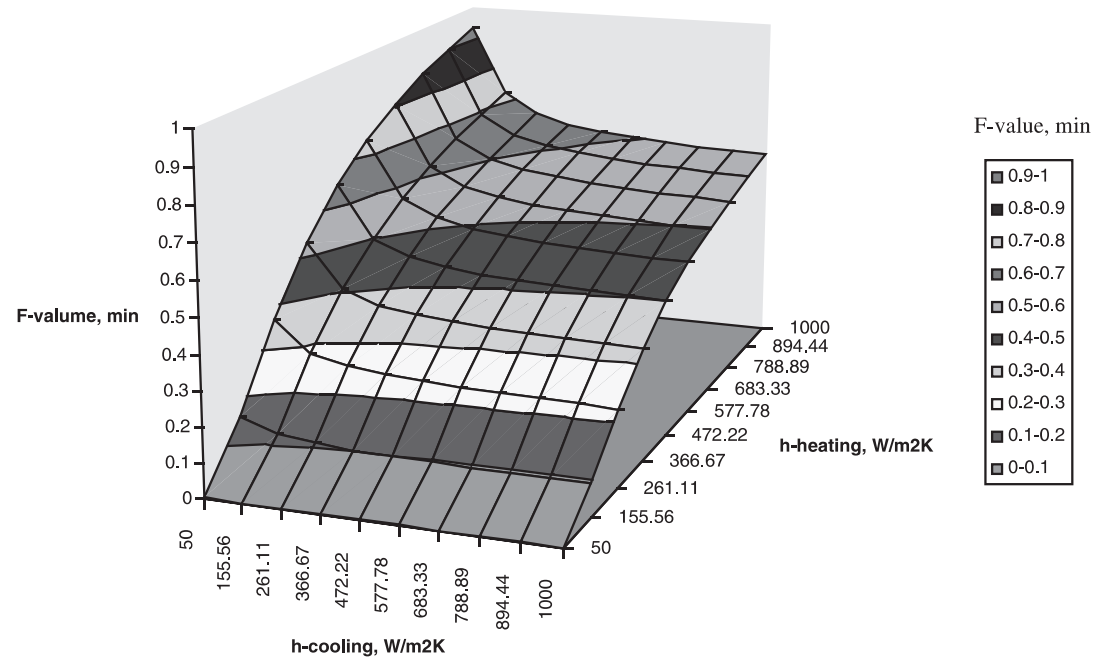

Fig. 6. Effect of the average heat transfer coefficients in heating and cooling on the lethality at the geometric centre of cans, for the thermal treatment of the first replicate with layout a.

heat transfer coefficient during heating has a more significant effect than that of cooling. At large values of $h_{\text {heat }}\left(>600 \mathrm{~W} / \mathrm{m}^{2} \mathrm{~K}\right)$ and small values of $h_{\text {cool }}$ $\left(<200 \mathrm{~W} / \mathrm{m}^{2} \mathrm{~K}\right)$ a small increase in the heat transfer coefficient for cooling resulted in a significant decrease in the $F$-value, but in that range $h_{\text {heat }}$ did not affect the lethality considerably. The lethality varied the most with respect to $h_{\text {heat }}$ when its magnitude was within the range $150-260 \mathrm{~W} / \mathrm{m}^{2} \mathrm{~K}$. Considering that the calculated heat transfer coefficients were within this range and $h_{\text {cool }}$ was always below $200 \mathrm{~W} / \mathrm{m}^{2} \mathrm{~K}$, Fig. 5 suggests that a small variability in the heat transfer coefficient during heating may affect the lethality distribution, while variations during cooling are less relevant.

\section{Conclusions}

The heat transfer coefficient in a retort can be calculated as a function of time by determining the average coefficient and derivating the result. It was found that the heat transfer coefficient increases sharply in the first few minutes and then remains relatively constant, increasing slightly. The use of constant heat transfer coefficients during heating and cooling was sufficient to describe very accurately the temperature histories inside conduction heating products, in the retort used. The results indicated that for the bentonite-containing cylindrical cans the average heat transfer coefficients were about $150-200 \mathrm{~W} / \mathrm{m}^{2} \mathrm{~K}$ for heating and 90-140 W/m $\mathrm{m}^{2} \mathrm{~K}$ for cooling. In this range, the variability of the heat transfer coefficient during heating affects the lethality delivered, which is in turn relatively insensitive to variability in the heat transfer coefficient during cooling.

\section{References}

Anantheswaran, R. C., \& Rao, M. A. (1985a). Heat transfer to model Newtonian liquid foods in cans during end-over-end rotation. Journal of Food Engineering, 4, 1.

Anantheswaran, R. C., \& Rao, M. A. (1985b). Heat transfer to model non-Newtonian liquid foods in cans during end-over-end rotation. Journal of Food Engineering, 4, 21

Azevedo, I. C. A., Oliveira, F. A. R., Drumond, M. C. S. (1998). A study on the accuracy and precision of external mass transfer coefficients jointly estimated from pseudo-experimental simulated data. Mathematics and Computers in Simulation, in press.

Burfoot, D., \& James, S. J. (1988). The effect of spatial variations of heat transfer coefficient on meat processing times. Journal of Food Engineering, 7, 41

Burfoot, D., \& Self, K. P. (1988). Prediction of heating times for cubes of beef during water cooking. International Journal of Food Science and Technology, 23, 247.

Chang, S. Y., \& Toledo, R. T. (1989). Heat transfer and simulated sterilization of particulate solids in a continuously flowing system. Journal of Food Science, 54, 1017.

Dickerson, Jr., R. W., \& Read, Jr., R. B. (1968). Calculation and measurement of heat transfer in foods. Food Technology, 22, 1533.

Hubbard, L. J., \& Farkas, B. E. (1998). Determination of the convective heat transfer coefficient during immersion frying. In Proceedings of the 11th International Drying Symposium (IDS '98), Halkidini, Greece, 19-22 August 1998, vol. A, 781-788.

Lebowitz, S. F., \& Bhowmik, S. R. (1989). Determination of retortable pouch heat transfer coefficients by optimization method. Journal of Food Science, 54, 1407.

Lebowitz, S. F., \& Bhowmik, S. R. (1990). Effect on retortable pouch heat transfer coefficients of different thermal processing stages and pouch material. Journal of Food Science, 55, 1421.

Ling, A. C., Lund, D. B., \& Lightfoot, E. N. (1976). Estimation of surface heat transfer coefficient with the presence of non-condensable gases. Journal of Food Science, 41, 1345.

McGinnis, D. S. (1986). Surface heat transfer distribution in a weir type pressurized water retort for processing foods in flexible retort pouches. Canadian Institute of Food Science and Technology Journal, 19, 45.

Merson, R. L., Stoforos, N. G. (1990). Motion of spherical particles in axially rotating cans. Effect on liquid-particle heat transfer. In 
W. E. L. Spiess, \& H. Schubert, Engineering and Food, vol. 2 (p. 160). Amsterdam, The Netherlands: Elsevier.

Naveh, D., \& Kopelman, I. J. (1980). Effect of some processing parameters on the heat transfer coefficients in a rotating autoclave. Journal of Food Processing and Preservation, 4, 67.

Ramaswamy, H. S., Tung, M. A., \& Stark, R. (1983). A method to measure surface heat transfer from steam/air mixtures in batch retorts. Journal of Food Science, 48, 900.

Ramaswamy, H. S., \& Tung, M. A. (1990). Heat transfer coefficients for water immersion heating media in vertical retorts. In W. E. L. Spiess, \& H. Schubert, Engineering and Food, vol. 2 (pp. 85-94). London: Elsevier.

Rumsey, T., Farkas, D. F., \& Hudson, J. S. (1980). Measuring the steam heat transfer coefficient to vegetables. Transactions of the ASAE, 1048.
Tucker, G. S., Holdsworth, S. D. (1991). Mathematical modelling of sterilisation and cooking processes for heat preserved foods. Application of a new heat transfer model. Transactions of the IChemE, Part C, 69, 5.

Varga, S., Oliveira, J .C., Smout, C., \& Hendrickx, M. (2000). Modelling temperature variability in batch retorts and its impact on lethality distribution. Journal of Food Engineering, 44, $163-174$.

Xie, G., \& Sheard, M. A. (1996). Comparison of two methods used to determine apparent heat transfer coefficient for pouches pasteurized in a combination oven. Food Science and Technology - LWT (Lebensmittel Wissenschaft und Technologie), 29, 100. 